\title{
Análise espacial da produtividade agrícola no Estado do Paraná: implicações para o seguro agrícola
}

\author{
Vitor Ozaki ${ }^{1}$
}

Resumo: O presente estudo tem como objetivo analisar espacialmente os dados de produtividade agrícola. Através da estatística espacial, é possível estimar alguns parâmetros do semivariograma relevantes para estudar o problema do risco sistêmico, com implicações nos programas de seguro agrícola. Em particular, estimaram-se os parâmetros de alcance para se verificar a distância, na qual a correlação espacial tende a zero. Para a análise empírica, utilizaram-se dados de produtividade agrícola municipal do IBGE para soja e milho, no estado do Paraná, no período de 1990 a 2002. $\mathrm{O}$ estudo mostrou que, de fato, a dependência espacial destes dados existe, tornando-se praticamente nula a distâncias relativamente longas (em $\mathrm{km}$ ) e podendo ser captada em todos os anos, para ambas as culturas.

Palavras-chave: seguro agrícola; risco sistêmico; correlação espacial; semivariograma.

Abstract: This paper analyses the spatial pattern of the agricultural yield data. Using the spatial statistics, it is possible to estimate some parameters of the semivariogram to study the problem of the systemic risk, which has great implications for the crop insurance program

${ }^{1}$ Professor Doutor do Departamento de Ciências Exatas - Matemática e Estatística da Esalq/USP. 
in Brazil. In particular, the "range" parameter was estimated. This parameter measures the distance, in which the spatial correlation tends to zero. For the empirical analysis, county yield data was used provided by IBGE, for soybean and corn, in the state of Paraná, through 1990 and 2002. The results showed that there is spatial dependence in every year analysed, going to zero in relatively long distances (in $\mathrm{km}$ ).

Key-words: crop insurance; systemic risk; spatial correlation; semivarioram.

\section{JEL Classification: Q19}

\section{Introdução}

A agricultura é uma atividade caracterizada pela vulnerabilidade a eventos que estão fora do controle do produtor. Notadamente, uma das principais causas da redução da produtividade agrícola são os eventos climáticos. Seca, geada, chuva excessiva, granizo e diversos agentes biológicos, entre outras adversidades, podem afetar diversas culturas em diferentes estágios de desenvolvimento.

Uma característica perversa da ocorrência desses eventos para os agricultores é quanto à extensão dos danos e ao tamanho dos prejuízos econômicos ${ }^{2}$. Em outras palavras, quando ocorre seca, por exemplo, este fenômeno não afeta apenas um único produtor, mas muitos e em uma vasta extensão territorial. Este fenômeno é conhecido como risco sistêmico ou risco de evento generalizado.

O risco sistêmico está diretamente ligado à presença de correlação espacial nos dados $^{3}$. Quando ocorre o fenômeno climático, a

\footnotetext{
${ }^{2}$ Por exemplo, o longo período de estiagem ocorrido entre o final de 2004 e início de 2005 provocou perdas consideráveis nos estados da região Sul, em Mato Grosso do Sul, São Paulo e Goiás, estimadas em 19 milhões de toneladas (perda de cerca de $25 \%$ da produção estimada) somente nas culturas de milho e soja. Os prejuízos aos agricultores são estimados em R $\$ 10$ bilhões (pouco mais de US $\$ 4$ bilhões).

${ }^{3}$ Para se detectar o impacto e a extensão da ocorrência de um evento climático adverso, seriam necessários dados de produtividade coletados em anos que de fato ocorreu este evento. Na ausência destes, dados mais agregados podem dar uma indicação da extensão territorial do risco sistêmico.
} 
extensão territorial dos danos causados pode ser detectada através do semivariograma, ou seja, uma função matemática que relaciona a correlação e a distância.

Nos EUA, a correlação espacial se reduz mais rapidamente com o aumento da distância, em anos regulares (sem a incidência de qualquer evento climático danoso), estabilizando-se no valor de 0,10 quando a distância entre as áreas consideradas é de aproximadamente $322 \mathrm{~km}$ (Goodwin, 2001).

Considerando anos em que secas ocorreram, a medida de correlação decresceu mais vagarosamente, atingindo o mesmo nível de 0,10 a uma distância de $644 \mathrm{~km}$. Em anos de ocorrência de eventos generalizados, o problema do risco sistêmico é mais acentuado que em anos normais.

Outro estudo a respeito da correlação espacial, utilizando correlogramas para a determinação da estrutura de dependência espacial, mostrou que a distância máxima estimada para a correlação não-negativa foi de $917 \mathrm{~km}$ (Wang e Zhang, 2003).

Dentro do contexto do mercado de seguros, o risco sistêmico é um dos maiores problemas enfrentados pelas seguradoras privadas no ramo rural (Miranda e Glauber, 1997).

O presente estudo tem como objetivo realizar inferências sobre a correlação espacial nos dados de produtividade agrícola municipais. Em particular, será quantificada a extensão territorial (em km), na qual a correlação espacial tende a zero. Para isso, serão utilizados semivariogramas, de modo que o parâmetro de alcance será o parâmetro de interesse na análise.

\section{Fonte de dados}

Os dados de produtividade municipal utilizados na pesquisa são provenientes do IBGE (Instituto Brasileiro de Geografia e Estatística), correspondentes ao período de 1990 a 2002, para as culturas do milho e da soja, no estado do Paraná.

O estado do Paraná é o maior produtor de milho no Brasil - totalizando 9,8 milhões de toneladas em 2002, pouco mais de $27 \%$ de toda produção brasileira - e o quarto maior em termos de produtividade média do milho (3.987 quilos por hectare) (IBGE, 2004). 


\section{Metodologia}

\subsection{Design do contrato de seguro agrícola}

A idéia do seguro é indenizar o produtor toda vez que a produtividade estiver em um patamar abaixo da garantida pelo contrato de seguro. Esse tipo de seguro é denominado seguro agrícola de produtividade. Basicamente, o mecanismo de compensação I para cada propriedade rural $i$ pode ser expressa da seguinte forma:

$$
I_{i}=\phi_{i} \max \left[\left(y_{i}^{c}-y_{i}\right), 0\right]
$$

Em que:

$\phi_{i}$ é a nível garantido ${ }^{4}, 0<\phi_{i}<1$;

$y_{i}^{c}$ é o nível de produtividade garantida e

$y_{i}$ é a produtividade observada.

A eq. (1) mostra que se a produtividade agrícola $y_{i}$ no final de determinado período for menor que a produtividade garantida $y_{i}^{c}$, então ocorre a indenização. Seu valor é igual a diferença entre a produtividade garantida e a produtividade observada ${ }^{5}$. Para isso, o segurado deve pagar um prêmio (preço do seguro) e obedecer determinadas requisições presentes na apólice (Ozaki, 2005).

Nesse tipo de contrato, o produtor tem a opção de escolher o nível de cobertura $\alpha_{i}$ que desejar, tal que $0 \leq \alpha_{i} \leq 1$. Dessa forma, a produtividade crítica é calculada de acordo com a equação: $y_{i}^{c}=\alpha_{i} \mu_{i}$, em que $\mu_{i}$ é a produtividade esperada do produtor $i$.

\subsection{Análise Espacial: O Método Geoestatístico}

A teoria das variáveis regionalizadas, ou geoestatística, foi inicialmente abordada em problemas relacionados à mineração na África do

\footnotetext{
${ }^{4}$ É igual a $(1-d)$, tal que $d$ representa a dedutibilidade. A dedutibilidade é o montante reduzido, em percentagem, do nível garantido (Spence e Zeckhauser, 1971; Pauly, 1974).

${ }^{5}$ Para se calcular o valor da responsabilidade por hectare (em termos monetários), deve-se multiplicar esta diferença por um preço determinado a priori.
} 
Sul e, mais tarde, na França. Matheron (1963) formalizou o corpo teórico dos métodos estatísticos que levavam em conta a estrutura da variabilidade espacial de determinado atributo. Como destaca Ribeiro Junior (1995):

“(...) cada observação é descrita não apenas pelo seu valor, mas também por informações de sua posição, expressa por um sistema de coordenadas. Considerando que observações mais próximas geograficamente tendem a ter valores mais similares e que tal fato pode ser avaliado por medidas de associação, fica claro que em geoestatística não se pode trabalhar com a suposição de independência."

Dessa forma, nota-se que o valor predito de uma variável leva em conta os valores dos atributos vizinhos, refletindo a dependência espacial das observações. Através das covariâncias e semivariâncias, os métodos geoestatísticos incorporam na análise tal estrutura de dependência.

Quando se consideram valores que apresentam um componente aleatório e certa estrutura de dependência espacial, a geoestatística é o método mais apropriado para a análise desses dados. Na literatura, diversos exemplos de aplicação do método podem ser citados, entre eles destacam-se estudos dos atributos do solo (como teor de minérios, fertilidade, nutrição, irrigação e drenagem) e de risco climático.

Conforme destaca Oliveira (2003), dois aspectos devem ser levados em conta na análise espacial. O primeiro se refere à modelagem da dependência espacial, considerando as pressuposições da distribuição Gaussiana e de estacionariedade e estimando os parâmetros através de métodos paramétricos, tais como método da máxima verossimilhança, verossimilhança perfilhada e através da abordagem bayesiana. O segundo leva em conta métodos não-paramétricos de estimação, como os métodos subjetivos (a sentimento).

No trabalho, serão abordados aspectos referentes à metodologia de análise da correlação espacial, entre eles estimação dos semivariogramas e parâmetros de alcance ${ }^{6}$.

${ }^{6}$ Do termo em inglês parâmetro range. 
Dessa forma, seja $y_{\mathrm{i}}$ determinado atributo representado pela produtividade agrícola, medido no local $x_{\mathrm{i}}$, define-se um processo espacial Gaussiano estacionário $\left\{\mathrm{Y}(x): x \in \mathfrak{R}^{2}\right\}$ como:

$$
\begin{aligned}
& \mathrm{E}[\mathrm{Y}(x)]=\mu, \forall \mathrm{x} ; \\
& \operatorname{Var}[\mathrm{Y}(x)]=\sigma^{2} ; \mathrm{e}, \\
& \rho^{e}(u)=\operatorname{Corr}\left[\mathrm{Y}\left(x_{i}\right), \mathrm{Y}\left(x_{j}\right)\right], \text { a função de correlação para } \mathrm{i} \neq \mathrm{j} .
\end{aligned}
$$

em que $u=\left\|x_{i}-x_{j}\right\|$ e corresponde a distância Euclideana entre $x_{i}$ e $x_{j}$ (Diggle e Ribeiro Jr., 2000). De forma complementar, define-se a covariância como:

$$
\operatorname{Cov}\left[\mathrm{Y}\left(x_{i}\right), \mathrm{Y}\left(x_{j}\right)\right]=\gamma^{e}\left(x_{i}-x_{j}\right)
$$

O processo será dito isotrópico ${ }^{8}$ se a covariância depender apenas da distância, ou seja, $\gamma^{e}\left(x_{i}-x_{j}\right)=\gamma^{e}\left\|x_{i}-x_{j}\right\|$. Desse modo, se o processo for estacionário, então $\gamma^{e}(u)=\sigma^{2} \rho^{e}(u)$. Se a variância puder ser expressa como Var $\left[\mathrm{Y}\left(x_{i}\right)-\mathrm{Y}\left(x_{j}\right)\right]=2 V\left(x_{i}, x_{j}\right)$, então, $2 V(\cdot)$ será denominada variograma (Matheron, 1963) e $V(\cdot)$, semivariograma.

Variograma é função matemática que representa a dependência entre duas variáveis aleatórias regionalizadas locais (Oliveira, 2003). Na figura 1, pode-se identificar os parâmetros do semivariograma: o efeito pepita $\tau^{2}$, o patamar $\sigma^{2}$ e o parâmetro de alcance $\phi^{e}$.

Nota-se que, neste trabalho, o parâmetro de interesse é apenas $\phi^{e}$ 9. Lembrando que:

$$
V\left(x_{i}, x_{j}\right)=\sigma^{2}\left(x_{i}\right)+\sigma^{2}\left(x_{j}\right)-2 \operatorname{Cov}\left(x_{i}, x_{j}\right)
$$

\footnotetext{
${ }^{7}$ Pode-se definir dois tipos de processos estacionários: Processos intrinsicamente estacionários, no qual $\mathrm{E}\left[\mathrm{Y}\left(x_{i}\right)-\mathrm{Y}\left(x_{j}\right)\right]=0$ e $\operatorname{Var}\left[\mathrm{Y}\left(x_{i}\right)-\mathrm{Y}\left(x_{j}\right)\right]=2 \gamma^{e}(u)$ e estacionários de segunda ordem, tal que $\mathrm{E}[\mathrm{Y}(x)]=\mu$ e $\operatorname{Cov}\left[\mathrm{Y}\left(x_{i}\right), \mathrm{Y}\left(x_{j}\right)\right]=\gamma^{e}(u)$. Nota-se que a classe dos processos intrinsicamente estacionários contém a classe dos processos estacionários de segunda ordem (Cressie, 1989).

${ }^{8}$ Diz-se que o comportamento de uma variável é isotrópico, se a variabilidade espacial dos valores é a mesma para todos os sentidos ou direções. Caso contrário, será denominado anisotrópico. Isso porque o semivariograma depende da direção em que é calculado.

${ }^{9}$ Em um contexto mais geral, em que o objetivo final é a Krigagem, todos os parâmetros devem ser estimados (Diggle e Ribeiro, 2000).
} 
Figura 1 - Semivariograma ilustrativo, com os respectivos parâmetros.

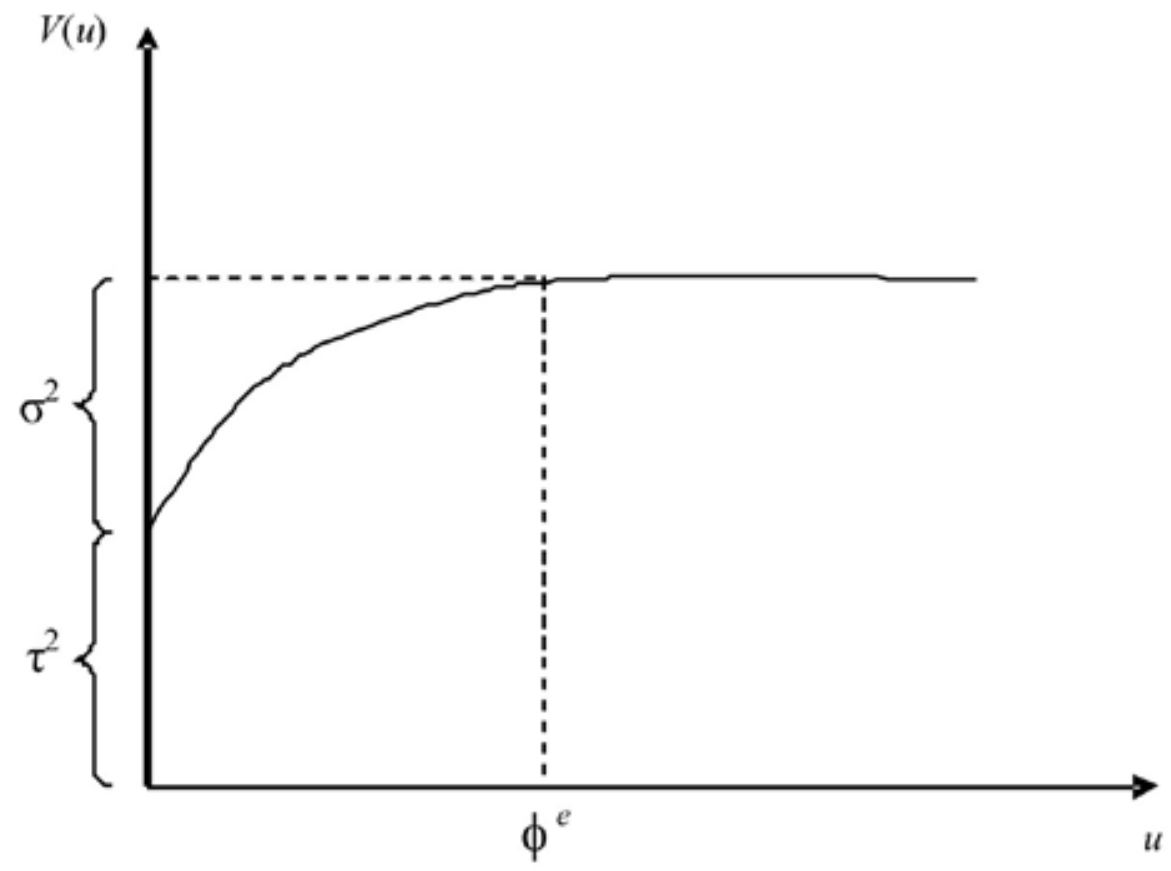

Então, se o processo for estacionário:

$V(u)=\sigma^{2}(x)\left[1-\rho^{e}(u)\right]$

Conforme aponta Cressie (1989), um estimador do variograma $2 V\left(x_{i}\right.$, $x_{j}$ ) que possa refletir a dependência espacial dos dados $\left\{\mathrm{Y}\left(x_{i}\right): i=1\right.$, $\ldots, n\}$ será dado por:

$$
\sum_{N(u)}\left[Y\left(x_{i}\right)-Y\left(x_{j}\right)\right]^{2} /|N(u)|
$$

conhecido como estimador empírico ou clássico.

Nota-se, porém, que o estimador empírico é sensível a outliers. Um estudo simulando a presença de outliers nos dados verificou que houve um impacto substancial na parte inicial do semivariograma, devido ao fato dos outliers se situarem perto dos dados de baixos valores (Diggle e Ribeiro, 2000). 
Conseqüentemente, isso pode gerar conclusões erradas a respeito da existência de dependência espacial e sobre o valor do efeito pepita ${ }^{10}$. Sendo assim, optou-se por utilizar, neste trabalho, um estimador mais robusto na presença de outliers, como o estimador proposto por Cressie e Hawkins (1980), dado por:

$$
\left\{(1 /|N(u)|) \sum_{N(u)}\left|Y\left(x_{i}\right)-Y\left(x_{j}\right)\right|^{1 / 2}\right\}^{4} /(0.457+0.494 /|N(u)|)
$$

A função de correlação está diretamente relacionada com o semivariograma. Diversas funções de correlação paramétricas podem ser incorporadas ao modelo. Entre elas, destacam-se: a família Esférica; a família Potência Exponencial ${ }^{11}$ e a família Matérn ${ }^{12}$ Cressie (1993). Como ilustração, considere a família Esférica:

$$
\rho^{e}\left(u ; \phi^{e}\right)=\left\{\begin{array}{l}
1-3 / 2\left(u / \phi^{e}\right)+1 / 2\left(u / \phi^{e}\right)^{3}, \quad 0 \leq u<\phi^{e} \\
0, \quad u \geq \phi^{e}
\end{array}\right.
$$

Nesta função, quando $u=\phi^{e}$ a correlação será igual a zero.

No trabalho, as séries de milho e soja foram analisadas no período de 1990 a 2002. Dessa forma, estimaram-se 26 semivariogramas empíricos, de acordo com o estimador robusto de Cressie e Hawkins, para todos os municípios do estado do Paraná em que havia dados nos respectivos anos.

Para estudar o comportamento da correlação espacial em cada um dos 26 anos, optou-se por analisar os semivariogramas, particularmente

${ }^{10}$ Do termo em inglês efeito nugget. O valor do efeito pepita é observado quando o semivariograma tende a zero.

${ }^{11} \rho^{\mathrm{e}}\left(u ; \phi^{e}, k\right)=\exp \left\{-\left(u / \phi^{e}\right)^{k}\right\}$, no qual $\phi^{e}>0$ e $0<k \leq 2$. Nestas funções, $k$ e $\phi^{e}$ são parâmetros, em que $k$ corresponde ao nível de alisamento do sinal $\mathrm{S}(x)$ e $\phi^{e}$ determina a taxa pela qual a correlação se aproxima de zero com o aumento da distância. Quando $k=1$, tal função recebe o nome de função de correlação Exponencial e quando $k=2$, função de correlação Gaussiana.

${ }^{12} \rho^{e}\left(u ; \phi^{e}, k\right)=\left\{2^{k-1} \Gamma(k)\right\}^{-1}\left(u / \phi^{e}\right)^{k} K_{k}\left(u / \phi^{e}\right)$. Nesta função $K_{k}(\cdot)$ representa a função Bessel do terceiro tipo de ordem $k$. 
através das estimativas do parâmetro de alcance $\left(\phi^{e}\right)$, obtidas por meio do método da máxima verossimilhança.

Se o processo for estacionário, então o semivariograma será dado por:

$$
V(u)=\sigma^{2}(x)\left[1-\rho^{e}(u)\right]
$$

Pode-se reescrever a equação anterior, de modo que:

$$
V(u)=\sigma^{2}(x)-\sigma^{2}(x) \rho^{e}(u)
$$

Representando, de forma geral, a correlação $\rho^{e}(\cdot) \operatorname{como} \gamma^{e}(\cdot) / \sigma^{2}$ $(x)$, então (Cressie, 1993):

$$
V(u)=\sigma^{2}(x)-\gamma^{e}(u)
$$

Caso $\gamma^{e}(u) \rightarrow 0$, quando $u \rightarrow \infty$, então a quantidade $\sigma^{2}(x)$ será denominada patamaris ${ }^{13}$.

Ajustou-se aos dados um modelo corrigido para a tendência (resultando em resíduos utilizados para a estimação do respectivo semivariograma) e outro modelo não-corrigido para a tendência. Para a escolha do melhor modelo, utilizou-se o critério "Bayesian Information Criteria" (BIC), que em termos de mudança do modelo $1\left(\mathrm{M}_{1}\right)$ para o modelo 2 $\left(\mathrm{M}_{2}\right)$ é dado por $-2 \log \left(\sup _{\mathrm{M} 1} \mathrm{f}(x / \theta)\right)\left(\sup _{\mathrm{M} 2} \mathrm{f}(x / \theta)\right)^{-1}-\left(\mathrm{p}_{2}-\mathrm{p}_{1}\right)(\log n)$.

Por meio da distribuição empírica dos dados, observou-se que boa parte das séries de milho apresentou certo grau de assimetria. Segundo Diggle et al. (2003), quando $\mathrm{Y}_{i}, \mathrm{i}=1, \ldots, n$, é contínua mas o ajuste de um modelo Gaussiano não parecer razoável, então a inclusão de um parâmetro extra $\lambda^{e}$ permite atingir certo grau de flexibilidade, definindo, dessa forma, uma transformação Box-Cox. Assim, os dados $\mathrm{y}_{i}$ podem ser transformados por meio de:

$$
y_{i}^{*}=\left(y_{i}^{\lambda^{e}}-1\right) / \lambda^{e}
$$

se $\lambda^{e} \neq 0$ e:

${ }^{13}$ Do termo em inglês sill. 


$$
y_{i}^{*}=\log y_{i}
$$

se $\lambda^{e}=0$.

Conseqüentemente, $y_{i}^{*}$ será a realização de um modelo Gaussiano. O parâmetro lambda foi estimado através do método da verossimilhança perfilhada. Neste ponto, ressalta-se que apenas três valores de lambda foram utilizados na transformação dos dados, de acordo com o intervalo de confiança a $95 \%$. São eles: lambda igual a zero (transformação logarítmica); igual a 0,5 (raiz quadrada) e igual a 1 (nenhuma transformação).

Na escolha entre os modelos com e sem tendência, através do critério BIC, os modelos sem tendência apresentaram-se mais adequados em todos os casos. Dessa forma, não foram utilizados os resíduos, mas os valores originais de produtividade na estimação dos semivariogramas.

Entre as diversas funções de correlação (5 para cada semivariograma, totalizando 130 semivariogramas) ajustadas aos modelos, optou-se pela função de correlação esférica, visto que o valor do logaritmo da função de verossimilhança (critério de seleção de modelos) ficou bastante próximo para as diferentes funções de correlação testadas para o cálculo do semivariograma de cada uma das 26 séries.

Neste estudo, estimaram-se os semivariogramas para diferentes direções, ou seja, para $0^{\circ}, 45^{\circ}, 90^{\circ}$ e $135^{\circ}$ (Diggle et al., 2003). Todas os semivariogramas estimados apresentaram um comportamento isotrópico.

\section{Resultados e discussão}

A dependência espacial foi diagnosticada, inicialmente, através da análise exploratória espacial, que fornece uma idéia geral do comportamento dos dados, levando-se em conta a localização desses dados no espaço. Além desta análise inicial, tal dependência pode ser verificada utilizando-se semivariogramas empíricos e envelopes simulados.

Desse modo, para que haja dependência, é desejável que os semivariogramas empíricos se comportem, de acordo com as figuras ${ }^{14} 2,3$,

${ }^{14}$ Nas figuras, as diferentes escalas no eixo vertical ocorrem devido às diversas transformações aplicadas aos dados iniciais. 
4 e 5, ou seja, o semivariograma aumenta a taxas decrescentes com distância, mas tende a se estabilizar em certo patamar.

Figura 2 - Semivariogramas ajustados para as séries de milho.
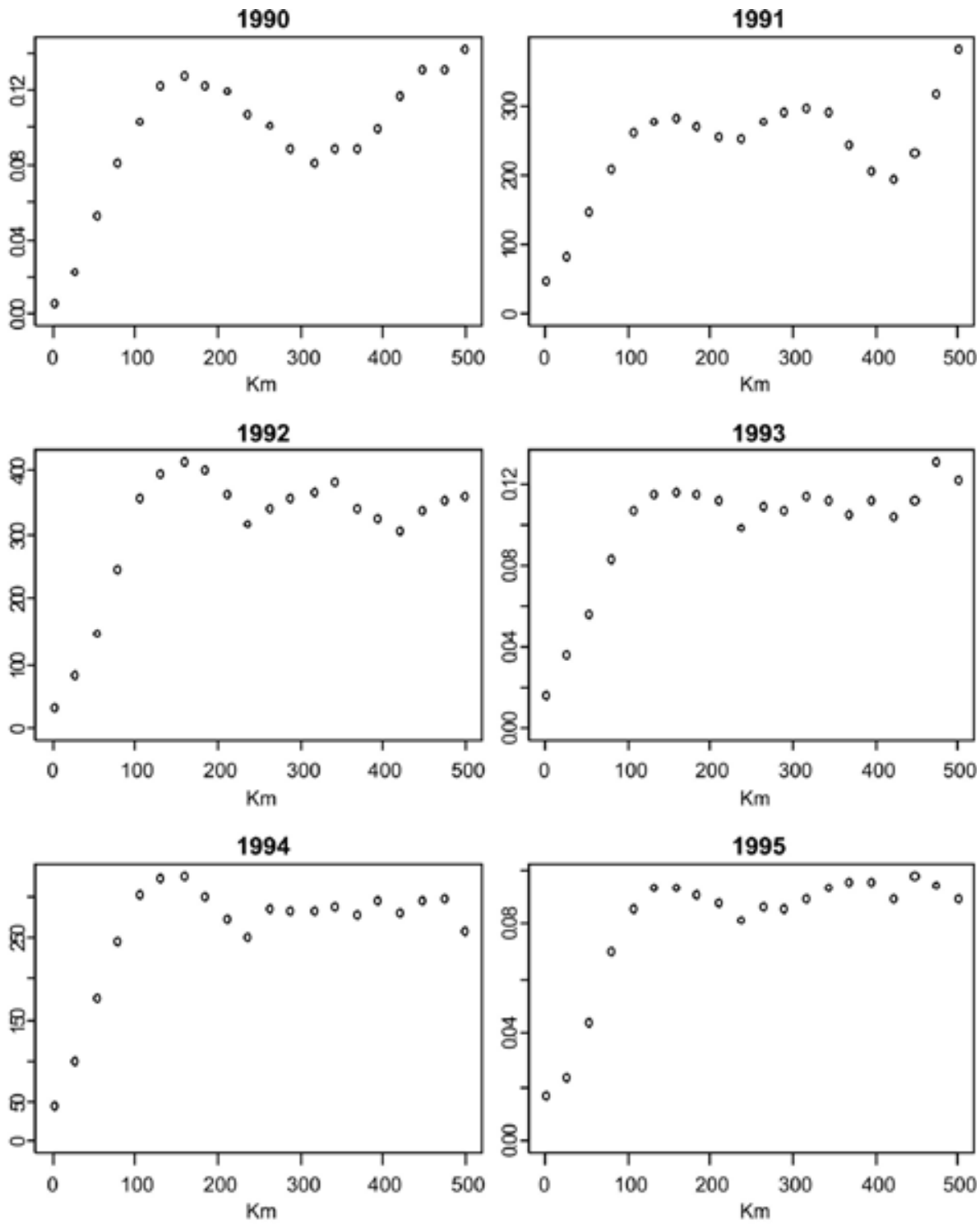

Fonte: Resultados da pesquisa. 
Figura 3 - Semivariogramas ajustados para as séries de milho.
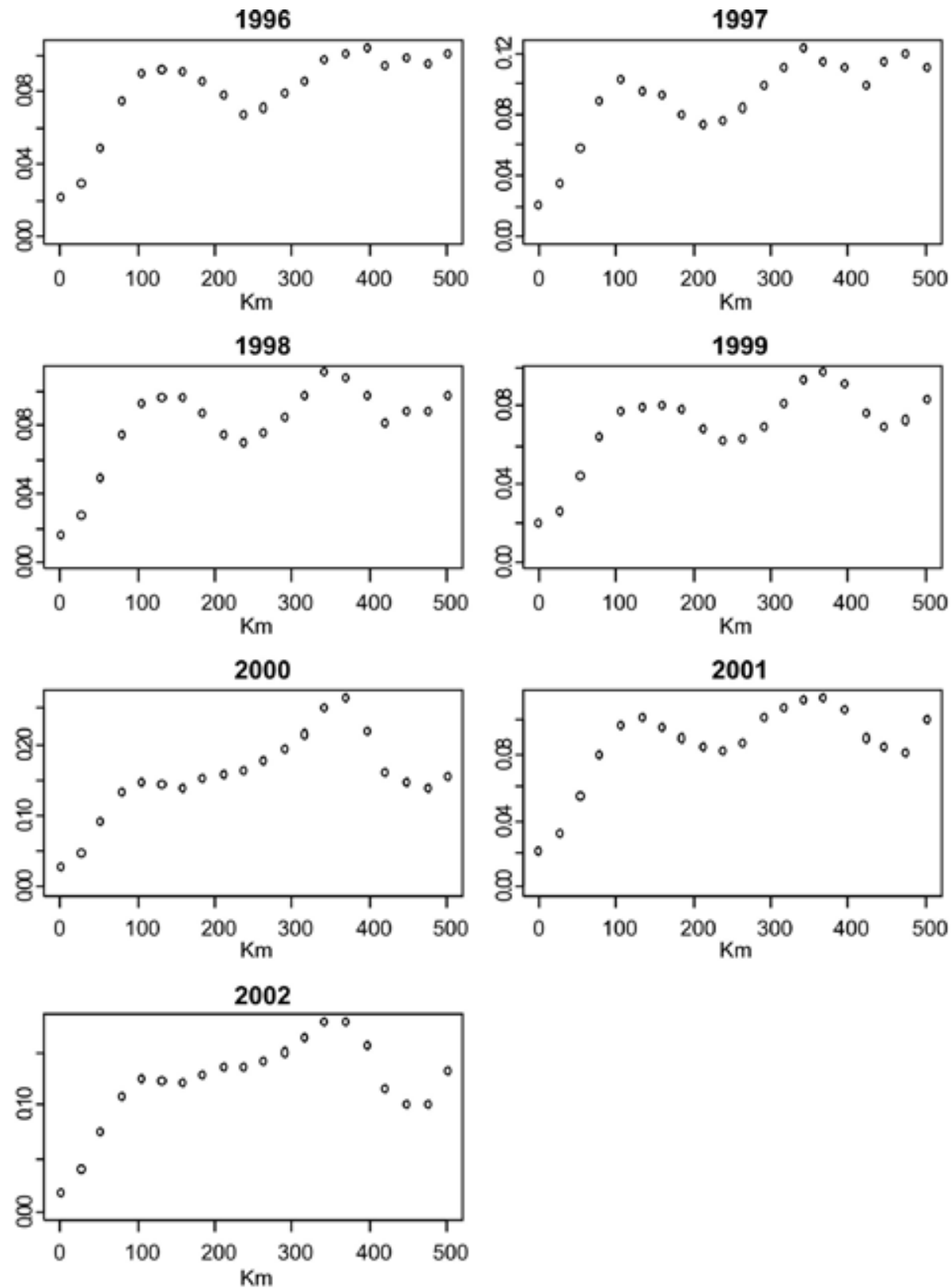

Fonte: Resultados da pesquisa. 
Figura 4 - Semivariogramas ajustados para as séries de soja.
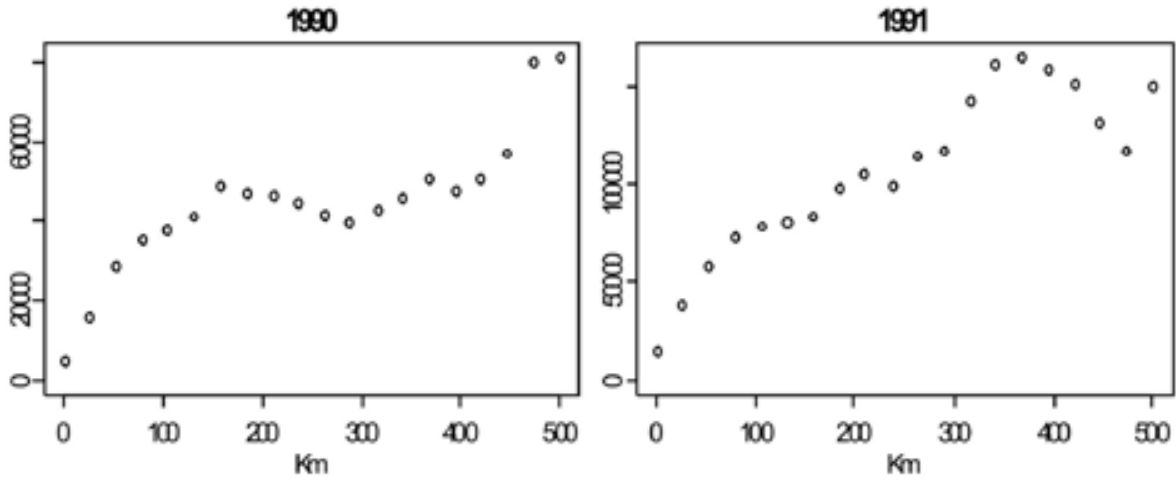

1998

1993
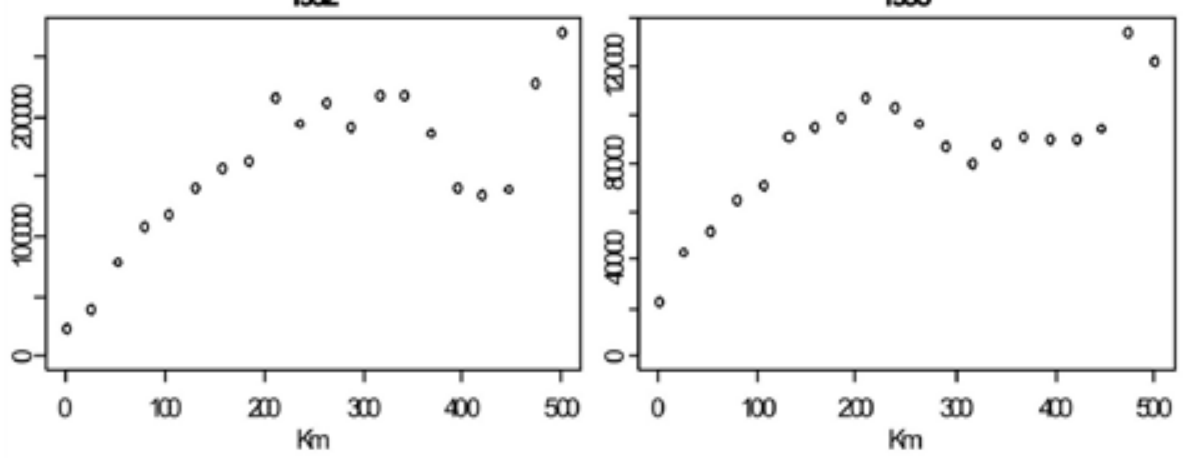

1994

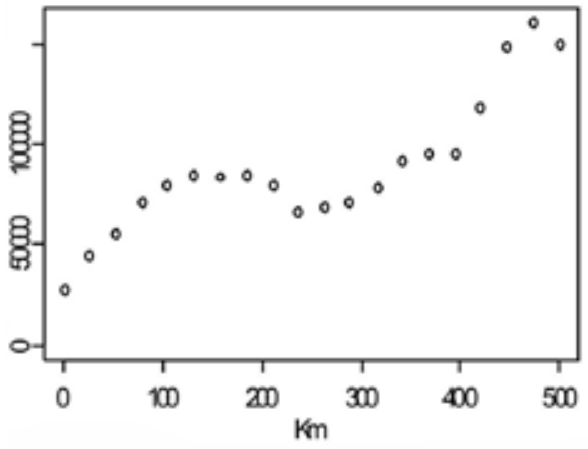

1996

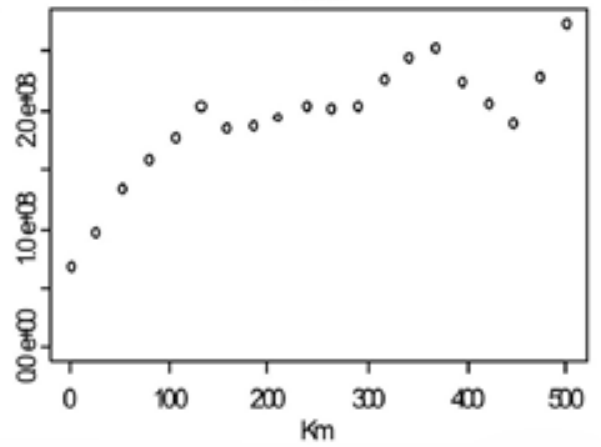

Fonte: Resultados da pesquisa. 
Figura 5 - Semivariogramas ajustados para as séries de soja.
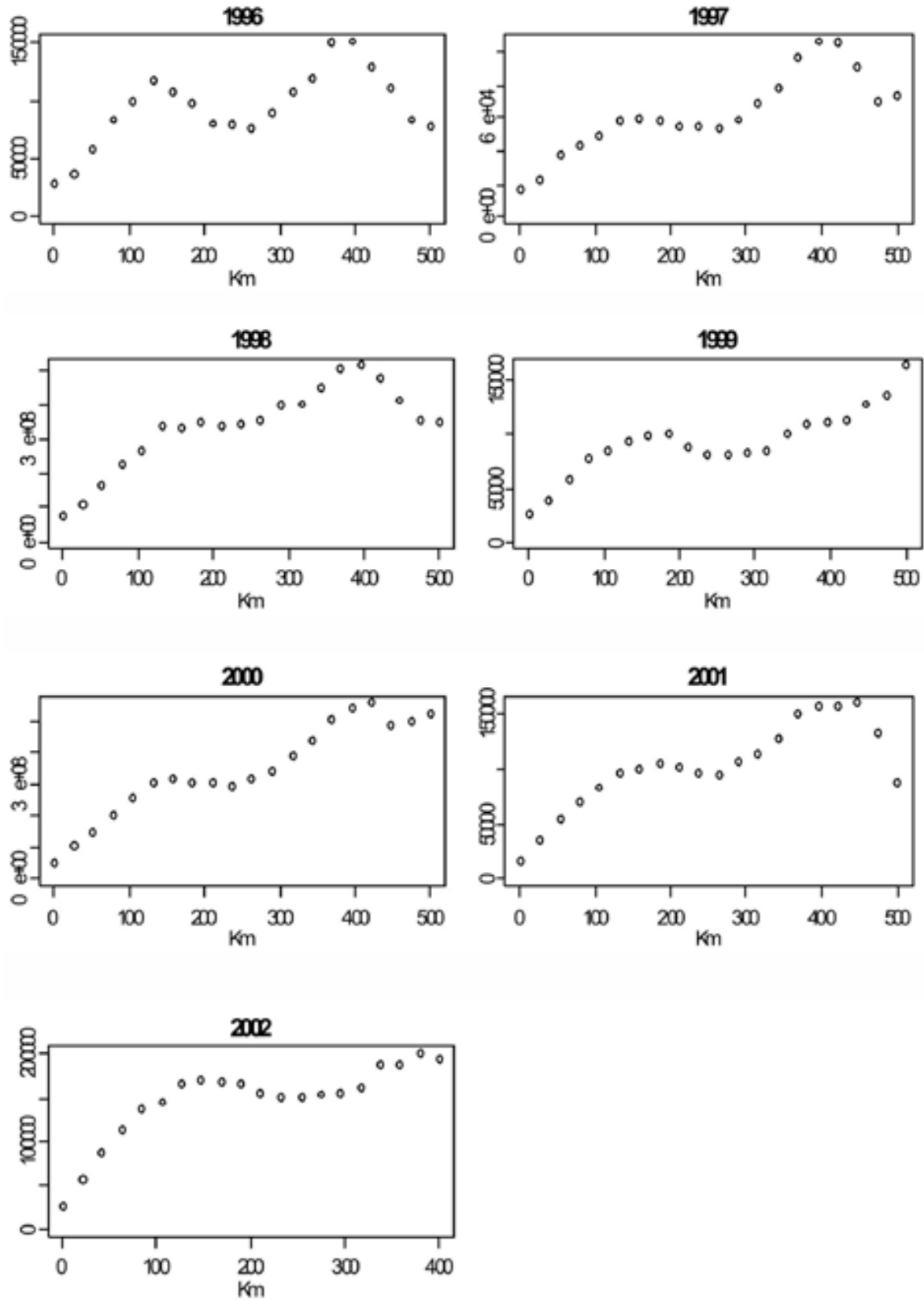

Fonte: Resultados da pesquisa. 
Na análise dos envelopes simulados ${ }^{15}$ (número de simulações igual a 99) deve haver ao menos um ponto do semivariograma fora do envelope (Diggle et al., 2003). Se isso ocorrer, rejeita-se a hipótese nula, de que não há dependência espacial. Nas simulações ${ }^{16}$, houve ao menos um ponto fora dos envelopes. Isso sugere a existência de correlação espacial nos dados de produtividade.

A tabela 1 mostra a distância na qual o semivariograma estabiliza em torno do patamar $\sigma^{2}(x)$. Percebe-se que, no caso do milho, dos 13 anos analisados, em nove a distância é menor do que na soja. Isso mostra que, para estes anos, a extensão da dependência espacial dos dados é, em média, igual a 132,4 km (milho) e 164,6 km (soja). Em outras palavras, a correlação espacial, no caso do milho, estabiliza a uma distância equivalente a $80 \%$ da soja, em média.

Tabela 1. Distância, em km, estimada pelo método da máxima verossimilhança, de 1990 a 2002, no estado do Paraná.

\begin{tabular}{ccc}
\hline Ano & Milho & Soja \\
\hline 1990 & 164,6 & 173,8 \\
1991 & 128,5 & 133,5 \\
1992 & 118,1 & 156,8 \\
1993 & 122,7 & 168,4 \\
1994 & 129,6 & 132,4 \\
1995 & 129,3 & 56,6 \\
1996 & 132,1 & 113,4 \\
1997 & 124,7 & 153,3 \\
1998 & 124,4 & 202,7 \\
1999 & 113,3 & 106,8 \\
2000 & 144,1 & 194,2 \\
2001 & 137,1 & 166,6 \\
2002 & 126,9 & 124,4 \\
média & 130,4 & 144,8 \\
\hline
\end{tabular}

Fonte: Resultados da pesquisa.

A mínima e a máxima distância ocorreram nos anos de 1999 e 1990, respectivamente, no caso do milho, e em 1995 e 1998, para a soja. Na

${ }^{15}$ Ver Diggle e Ribeiro (2000) para mais detalhes.

${ }^{16}$ Disponibilizado sob requisição ao autor. 
média, a distância na qual a covariância tende a zero é de aproximadamente 130 e de $145 \mathrm{~km}$, para o milho e a soja, respectivamente.

\section{Conclusões}

Os resultados mostram que, de fato, existe um padrão de dependência espacial nos dados, ou seja, as unidades seguradas não podem ser consideradas independentes espacialmente.

Esse fato tem repercussões perversas no mercado de seguros agrícola, tendo em vista que o risco de inadimplência das seguradoras perante os segurados é grande, na ocorrência do evento climático extremo. As seguradoras que operam neste mercado têm de estar atentas a estratégias de diversificação geográfica, evitando qualquer concentração de riscos em determinada região. Ademais, é fundamental a cobertura parcial ou total de resseguradoras e, também, algum outro tipo de cobertura contra riscos catastróficos, por exemplo, o FESR.

Atualmente, o mercado ressegurador e o FESR estão enfrentando mudanças. O primeiro está passando por um processo de abertura gradual e o segundo está sendo reestruturado por um grupo formado pelo IRB-Brasil Re, Susep e Ministério da Agricultura. Salienta-se que, sem a presença desses tipos de cobertura contra riscos catastróficos, o desenvolvimento e a sustentabilidade do mercado de seguro agrícola ficam comprometidos.

Na formulação de um programa de seguro em larga escala, o governo, seguradoras e resseguradoras poderiam, além da diversificação geográfica, diversificar suas atividades em outras modalidades do ramo rural, por exemplo: pecuário, benfeitorias e produtos agropecuários, penhor rural, florestas, vida, entre outros. A diversificação setorial garantiria, além do resseguro e do Fundo, uma maior segurança contra a ocorrência de eventos generalizados.

Futuras pesquisas podem se concentrar na análise espacial das propriedades agrícolas. Tomando-se como base um conjunto de propriedades em determinada região, pode-se utilizar os dados de produtividade com o objetivo de interpolá-los, resultando em mapas de predição para a região. Esses mapas poderiam ser de grande utilidade na verificação da produtividade esperada, variável essencial para a precificação de contratos de seguro agrícola. 
Os autor agradece à Coordenadoria de Aperfeiçoamento de Pessoal de Nível Superior (Capes) pelo apoio financeiro e aos comentários e sugestões de Rodolfo Hoffmann. Eventuais erros e omissões é responsabilidade unicamente do autor.

\section{Referências bibliográficas}

CRESSIE, N.A.C. Geostatistics. The American Statistician, v.43, n.4, p.197-202, Nov. 1989.

CRESSIE, N.A.C. Statistics for spatial data. New York: John Wiley \& Sons, 1993. 900p.

CRESSIE, N.A.C.; HAWKINS, D.M. Robust estimation of the variogram, I. Journal of the International Association for Mathematical Geology, v.12, p.115-125, 1980.

DIGGLE, P.J.; RIBEIRO JUNIOR, P.J. Model-based geostatistics. Caxambu: ABE, 2000. 129p.

DIGGLE, P.J.; RIBEIRO JUNIOR, P.J; CHRISTENSEN, O.F. An introduction to model-based geostatistics. In: MOLLER, J. Spatial statistics and computational methods. New York: Springer Verlag, 2003. 216p.

GOODWIN, B.K. Problems with market insurance in agriculture. American Journal of Agricultural Economics, v.83, n.3, p.643-649, Aug. 2001.

INSTITUTO BRASILEIRO DE GEOGRAFIA E ESTATÍSTICA (IBGE). www.ibge.gov.br. (julho, 2004).

MATHERON, G. Principles of geostatistics. Economic Geology, v.58, p.1246-1266, 1963.

MIRANDA, M. J.; GLAUBER, J. W. Systemic risk, reinsurance, and the failure of crop insurance markets. American Journal of Agricultural Economics, v. 79, n. 1, p. 206-215, fev 1997.

OLIVEIRA, M.C.N. Métodos de estimação de parâmetros em modelos geoestatísticos com diferentes estruturas de covariâncias: uma aplicação ao teor de cálcio no solo. Piracicaba, 2003. 140p. Tese 
(Doutorado) - Escola Superior de Agricultura "Luiz de Queiróz”, Universidade de São Paulo.

OZAKI, V.A. Métodos atuariais aplicados à determinação da taxa de prêmio de contratos de seguro agrícola: um estudo de caso. Piracicaba, 2005. 324p. Tese (Doutorado) - Escola Superior de Agricultura "Luiz de Queiroz”, Universidade de São Paulo.

PAULY, M. V. Overinsurance and public provision of insurance: the roles of moral hazard and adverse selection. Quarterly Journal of Economics, v. 88, n. 1, p. 44-62, fev. 1974.

RIBEIRO JUNIOR, P.J. Métodos geoestatisticos no estudo da variabilidade espacial de parâmetros do solo. Piracicaba, 1995. 99p. Dissertação (Mestrado) - Escola Superior de Agricultura "Luiz de Queiroz", Universidade de São Paulo.

SPENCE, M.; ZECKHAUSER, R. Insurance, information, and individual action. American Economic Review, v.61, n.2, p.380-387, May 1971. WANG, H.W.; ZHANG, H. On the possibility of private crop insurance market: a spatial statistics approach. The Journal of Risk and Insurance, v.70, n.1, p.111-124, 2003. 